\title{
The International System of Units-a case for reconsideration
}

\author{
Ulrich Feller
}

Received: 14 October 2010/Accepted: 27 December 2010/Published online: 30 January 2011

(C) The Author(s) 2011. This article is published with open access at Springerlink.com

\begin{abstract}
The International System of Units (SI) follows a concept that goes back to Maxwell. At that time, a logic sound foundation of mathematics was not yet available. This has lead to concepts and terms that are contradictory and in conflict with today's standard mathematical concepts. The inconsistencies that have evolved in metrology due to the lack of appropriate notions are pointed out. This is most important, as the metrology is a field that is internationally well organized under the umbrella of the Meter Convention, an international treaty for acting on all matters relating to units of measurement. Committees and working groups under the Meter Convention have a leading role in the elaboration of important metrological guides, among others the International Vocabulary of Metrology. Therefore, it is highly desirable that their publications use well-founded concepts and terminology. It is consensus that it is desirable to find a system of units on invariant properties of nature and not on human artifacts, e.g., the prototype of the kilogram. However, the current proposals to improve that are in conflict with standard scientific concepts. It is shown in the paper how these inconsistencies can be avoided. The argumentation is based on the interpretation of numbers developed by mathematicians like Cantor, Dedekind, Peano, and others that have led the logic foundation of mathematics with set and number theory. This foundation excludes dogmas that have been forwarded
\end{abstract}

Disclaimer: The views expressed in this paper are those of the author only. They do not represent an official view or position of the Swiss Federal Office of Metrology.

U. Feller $(\bowtie)$

Federal Office of Metrology METAS,

3003 Berne, Switzerland

e-mail: ulrich.feller@bluewin.ch in the last century under the umbrella of the Meter Convention.

Keywords System of units - System of quantities . Quantity calculus · Base unit · Derived unit · Number . Quantity · Measurement · Dimension · Constant of nature

\section{Introduction}

The International System of Units (SI) follows a concept that goes back to Maxwell. At that time, a logic sound foundation of mathematics was not yet available. This has led to concepts and terms that are contradictory and in conflict with today's standard mathematical concepts.

The inconsistencies that have evolved in metrology due to the lack of appropriate definitions of concepts are pointed out. This is most important, as the metrology is a field that is internationally well organized under the umbrella of the Meter Convention [1], an international treaty for acting in common accord on all matters relating to units of measurement. Committees and working groups under the Meter Convention as well as other international organizations have a leading role in the further development of the SI and participate in the elaboration of important metrological guides, among others the International Vocabulary of Metrology (VIM) [2-4]. Therefore, it is highly desirable that their publications use clear concepts and terminology.

It is consensus that it is desirable to put up a system of units on invariant properties of nature and not on human artifacts like the prototype of the kilogram. However, the current discussions have brought up proposals to redefine other units as well, among others the candela and the mole. It is shown in this paper that these further proposals are in 
conflict with standard scientific concepts and how these inconsistencies can be avoided.

The argumentation is based on the interpretation of numbers developed by mathematicians like Cantor, Dedekind, Peano, and others that have led the logic foundation of mathematics with set and number theory. Whenever we express knowledge in mathematical language, we apply always the same mathematics, independent of whether we work in pure mathematics, natural sciences, economy, psychology, or any other science. This presumption, however, has far-reaching consequences and excludes dogmas that have been forwarded in the last century under the umbrella of the Meter Convention.

\section{Numbers and quantities}

The creation of numbers is one of the greatest achievements of mankind. It has enabled to express experience with the animate and inanimate nature in an unambiguous way that can be communicated to other people, who can check in turn themselves the truthfulness of the information gained by this experience. Numbers are at the basis of mathematical concepts such as "variable" or "quantity" and "function" which enable to express relations between observable phenomena in an abstract logical language, as the laws of nature. In this paper, the term "phenomenon" is used as a generic term for object, state, or process in the observable world.

The nature of the term "number" has been the subject of intense research and discussion in the nineteenth century. Famous mathematicians like Cantor, Dedekind, Peano, and others have finally led the logical foundation for numbers in set and number theory. In these theories, the relationship between elements of a set and the concept of numbers has been worked out and the meaning of numbers has been put on a logical sound basis. Numbers have been classified depending on their properties in natural, integer, rational, real, and other types of numbers. Richard Dedekind has written in 1888 his famous text on The Nature and Meaning of Numbers, where he states [5]:

Numbers are free creations of the human mind; they serve as a means of apprehending more easily and more sharply the difference of things. It is only through the purely logical process of building up the science of numbers and by thus acquiring the continuous number-domain that we are prepared accurately to investigate our notions of space and time by bringing them into relation with this numberdomain created in our mind.

This means numbers are purely abstract concepts of our thinking, and when we use numbers, they are of the type as defined in mathematics: integer, rational, real, etc., numbers.

Many statements are valid for many different sets of numbers, so we use quantities (or-in the mathematical language-variables) for expressing statements valid for whole ranges of numbers. In mathematics, all quantities have an associated domain, i.e., a set of input values on which the quantity is defined. The relations among the numbers are then expressed as functions between quantities. Quantities as used in sciences are therefore generalized forms of numbers. They stand in place of whole sets of numbers; in other words, quantities are placeholders for numbers.

Two facts emerge from these mathematical concepts:

Firstly, if numbers are free creations of the human mind, quantities as generalized forms of numbers are free creations of the human mind, too. Quantities do not exist in nature, cannot be observed in nature, and quantities are abstract mathematical concepts we use for describing nature. The relation of the mathematical objects "quantity" to observable phenomena is described in the next paragraph.

Secondly, the values of quantities are numbers, as defined in mathematics: natural, integer, real, etc., numbers. With quantities, we perform mathematical operations and calculations, for example in the form of an equation like $F=m \cdot a$. In this well-known second law of Newton, the quantities $F, m$, and $a$ are mathematical variables defined on a domain or codomain of numbers. For the mathematical concepts underlying numbers, variables and functions, the reader is referred to the vast mathematical literature $[6,7]$.

These logically well-founded mathematical concepts exclude definitions for "quantity" and "value" as given in the 2008 VIM [4] where "quantity" is defined as follows:

\section{quantity}

property of a phenomenon, body, or substance, where the property has a magnitude that can be expressed as a number and a reference.

In the preceding editions of the VIM published in 1983 [2] and 1993 [3] and in many publications adopting the terminology of the VIM, the more familiar term "unit" is used instead of "reference" and the value of a quantity is expressed as the product of a number and a unit. The calculation with such products is called quantity calculus [8].

Mathematics tells us that quantities are free creations of the human mind and not properties of phenomena. Mathematical operations are defined only with quantities, not with properties. Mathematics tells us further that the values of quantities are numbers: natural, integer, real, etc., numbers. There is no mathematical foundation for values 
other than the well-known sets of numbers. If the values of quantities are expressed as products of numbers and units, logically well-founded mathematical concepts are mixed up with non-mathematical things like units. This must lead unavoidably to logical conflicts, as we will see below.

As postulated in the introduction, the mathematical concepts applied in whatever science are the same as in pure mathematics, and there is no need for a "quantity calculus" based on products of numbers and units. Therefore, there is no mathematical textbook that conveys this concept.

These statements are not at all of academic interest only. On the contrary, unclear definitions of terms and concepts of mathematical and observable things prevent clear and understandable concepts and definitions in physics and other sciences and lead to logical conflicts.

\section{Measurements}

If the mathematics as taught in the standard mathematical literature and the mathematics as applied in other fields of science is the same, the question arises what the difference is between a purely mathematical variable and a quantity as used in other sciences. Also for this question, Dedekind delivers the key. The quotation cited earlier continues:

If we scrutinise closely what is done in counting an aggregate or number of things, we are led to consider the ability of the mind to relate things to things, to let a thing correspond to a thing, or to represent a thing by a thing, an ability without which no thinking is possible.

Applied to sciences dealing with observable phenomena, this means the following: When we observe or measure phenomena, we count an aggregate or number of things. This way, we get a number for the measured quantity. This number represents the observed thing/phenomenon. Thus, we relate things of our imagination (values/numbers of the measured quantity) to things in the observable world and let this way a thing (value) correspond to a thing (observed phenomenon). Quantities and numbers are related to-and correspond to-observable phenomena.

An instructive example of this principle is given by Richard Feynman in his famous lectures on physics, where he discusses the meaning of the term "energy" [9]:

To illustrate the ideas and the kind of reasoning that might be used in theoretical physics, we shall now examine one of the most basic laws of physics, the conservation of energy. ... It states that there is a certain quantity, which we call energy, that does not change in the manifold changes which nature undergoes. That is a most abstract idea, because it is a mathematical principle; it says that there is a numerical quantity which does not change when something happens. It is not a description of a mechanism, or anything concrete; it is just a strange fact that we can calculate some number and when we finish watching nature go through her tricks and calculate the number again, it is the same.

This example illustrates the correspondence between number and observable world, the correspondence being established by measurement.

In today's language, the answer to the question above can therefore be formulated as follows: For quantities we use in science to describe, model, or simulate mathematically observable phenomena, there are well-specified measurement procedures. It does not matter whether the quantity describes a number of apples or eggs in a packaging, the national income of a country, the mass of an object, customer satisfaction, electrical voltage, or any other quantity.

For each measurable quantity, there exists one or more specific measurement procedures that distinguish this quantity from other quantities. Thus, the number of apples is measured differently than the national income, this in turn differently from the mass of an object, and so on. At the end of the measurement, we have a number, or,considering the measurement uncertainty-a range of numbers that corresponds to the thing that is measured.

The only difference between the measurements of different things is that mankind has developed in the course of time more or less sophisticated procedures for counting. Thus, the "measurement" of apples or bananas is performed by simple counting. The measurement of customer satisfaction or the national income requires more sophisticated counting. In chemistry, we have a marvelous instrument at hand for counting the number of atoms or molecules [10], the balance, and for measuring physical properties, transducers have been developed that convert the observed phenomenon/thing into a countable signal. At the end of every measurement, the numbers are obtained either by simple counting, by counting the marks on an analog scale, or by counting the pulses of an analog to digital converter. In even more complicated situations, the counted numbers are to be processed further by calculation, as in energy measurement, where no direct measurement procedure exists, as explained beautifully in the lecture of Richard Feynman cited earlier.

Quoting Dedekind once more [5], quantities are free creations of the human mind, they do not exist in nature. Quantities depend on how we interact with nature, as they are related to specified measurement procedures as explained earlier. If we are interested in the number of 
apples, we simply count them. If we are interested in the mass of apples, we put them on a balance. If we are interested in the spatial extension/size of apples, we take a ruler or a caliper rule, or we apply volt- and amperemeter, when we are interested in the conductivity of the flesh of the apple. The object of measurement in this enumeration of actions is always the same, an apple. But the way how we interact with it in measurement varies from quantity to quantity we are interested in.

Thus, measurable quantities are defined by the manner how we interact with the observable world, the interaction being called measurement. They are not given by nature, thus, are not "properties" of observable phenomena as stated in the VIM. Measurable quantities "correspond" or "represent" properties of observable phenomena, as Dedekind expresses it. This distinction between abstract mathematical concepts and observable world is essential for a clear understanding of how science works.

\section{Units}

When we communicate measurement results, we have to state clearly to what quantities the numbers belong and what units of measurement were chosen. When we count apples, the number is reported in the unit "apple". When we measure national income, the result is indicated in "Euro" (symbol €) or "dollar" (symbol \$), and when we report on the measurement of mass or current in the SI, we say that the unit of measurement was the "kilogram" (symbol kg) or "ampere" (symbol A).

The quotation marks are set because neither "apple" nor "Euro" (€) nor "kilogram" (kg) nor "ampere" (A) are units. They are names (symbols) of units. These names and symbols are used for expressing what things of the observable world were considered in measurement. They are not part of the mathematics (quantities and numbers) that corresponds or represents the observed thing. As explained earlier, the values of quantities are pure numbers, as defined in mathematics (natural, integer, real numbers, etc.). The mathematical literature does not provide units as used in counting or measurement. The names or symbols of units appended to the numbers for pointing to the units used in measurement are part of the descriptive language and are not mathematical objects. In particular, the names and symbols of units have neither "magnitude" nor "dimension".

With units we make measurements, not mathematics: Units are anything but free creations of the human mind, hence not quantities, in contrast to the definition given in the VIM and in many standards that adopt the definitions of the VIM. In the VIM, unit is defined as follows [4]:

\section{measurement unit}

unit of measurement

unit

real scalar quantity, defined and adopted by convention, with which any other quantity of the same kind can be compared to express the ratio of the two quantities as a number

In this phrase the free creation of the human mind, "scalar quantity" is confounded with the observable thing "unit", which can be compared with another observable thing of the same kind. No distinction is made between the abstract mathematical objects corresponding to the observed phenomena, and the phenomena themselves. Furthermore, it is obvious that most units cannot be defined and adopted by convention. Once the system of units is fixed by suitable constants, most other units are fixed by the laws of nature as well.

The unit of a mathematical object is the unity element of the mathematical structure under consideration. For scalar quantities for example, this is the number 1 . The unit of measurement of a measurable quantity instead is an observable phenomenon/thing providing the value 1 of the quantity when measured. The unit, when realized according to its definition, represents the value 1 of the quantity and serves to gauge/adjust the measuring equipment for the quantity under consideration. Thus, the unit of mass is the PtIr-cylinder stored in a safe of the International Bureau of Weights and Measures (BIPM) near Paris, and is not "kilogram" or "kg". The ampere is a well-defined flow of charged particles and the volt an electrical state that would produce the reading $1.00000 \ldots$ on a perfectly calibrated instrument. Thousand real volts can kill a human, 1000 volts as indicated on the reading of a voltmeter or written in a publication kill nobody. Thus, a unit is anything but a quantity.

The mixing of pure mathematical concepts (numbers, quantities), practical actions in measurement (processes in the observable world) and elements of normal language (unit names, unit symbols) finds its roots apparently in a statement that is found in Maxwell's famous 1873 Treatise on Electricity and Magnetism. The quotation is taken from the comprehensive presentation of the subject by de Boer [8]:

Every expression of a quantity consists of two factors or components. One of these is the name of a certain known quantity of the same kind as the quantity to be expressed, which is taken as a standard of reference. The other component is the number of times the standard is to be taken in order to make up the required quantity. The standard quantity is technically called the unit and the number is called the numerical value of the quantity. 
de Boer continues: This may be expressed in the following notation: physical quantity $=$ numerical value $\times$ unit, $\mathrm{Q}=\{\mathrm{Q}\} \cdot[\mathrm{Q}]$.

It is not known to the author of this article whether Maxwell has understood his statement in the mathematical form as expressed by de Boer. But it is obvious that in this interpretation free creations of the human mind (quantity, number) are mixed with names and symbols used in normal language for denominating the observable things (units), and, both, the mathematical things (quantity) and the observable things are called "quantity". Mixed constructs consisting of mathematical and linguistic elements as expressed in the equation earlier have never found a logically correct foundation in mathematics and are not used there. It is easily understandable that this muddle of different things in mathematical expressions is a source of continuous logical problems, as is visible in the long-lasting discussions and publications on the subject [8]. The unsettled comprehension of the subject finds its counterpart in the VIM where the basic definitions in chapter 1 change from edition to edition [2-4].

Appreciating the historical evolution, it is to be noted that the logical foundations of mathematics with set and number theory had been achieved later in the nineteenth century by mathematicians like Cantor, Dedekind, Peano and were hardly known to Maxwell.

As an illustration for the confusion between mathematical expressions and names of units, even in the responsible bodies of the Meter Convention, the following statement in a recent report of the Consultative Committee for Units (CCU) is given, commenting the relation between the unit mole and the Avogadro constant $N_{\mathrm{A}}$ [11]:

For example, we define the mole by fixing the numerical value of $N_{A}$ when the value of $N_{A}$ is expressed in the SI unit inverse mole, thereby defining the mole, but the value of $N_{A}$ does not change. If, for example, we were to choose a larger number for the numerical value of $N_{A}$ we would be effectively defining a larger value for the unit mole, but the value of $N_{A}$, which is the numerical value multiplied by the unit inverse mole, would be unchanged.

This statement shows how the mere name of the unit mole is filled with mathematical content. It is said that the value of $N_{\mathrm{A}}$ does not change if the numerical value of $N_{\mathrm{A}}$ changes! Obviously, the unit symbol of $N_{\mathrm{A}}, \mathrm{mol}^{-1}$, in words "per mole", is considered as a mathematical object, carrying magnitude. As explained earlier, a unit name/ symbol does not bear any quantitative meaning, it is part of the normal language.
If we would allow—as a "Gedankenexperiment"-that also the name/symbol of the unit bears magnitude, the statement of the CCU would be:

$N_{\mathrm{A}}=\left\{N_{\mathrm{A}}\right\}$ per mol. Since a mol is $\left\{N_{\mathrm{A}}\right\}$-particles, the equation would read:

$N_{\mathrm{A}}=\left\{N_{\mathrm{A}}\right\}$ per $\left\{N_{\mathrm{A}}\right\} \cdot$ particles $=1$ per particle. The Avogadro constant would indeed be constant, irrespective of the numerical value of it, namely 1 (per particle). That is hardly what CCU wanted to express. But this example shows what bizarre results are achieved if mathematical, observable and linguistic things are not correctly kept apart, as Dedekind claims it. This example shows further that a logically sound description of nature based on mathematically well-founded concepts is urgent. In this description, mathematically not well-founded dogmas like physical quantity $=$ numerical value $\times$ unit must be abandoned!

\section{Constants}

What is a constant? In the mathematical sense the term "constant" is usually used for a number (integer, rational, irrational, etc.), the number having a proper name or symbol (dozen, $\pi$, e, ...). Hence, it can also be considered as a quantity with a fixed value. In sciences and technology, a constant stands mostly for a stable artifact or natural phenomenon which gives the known value when measured. Expressed in Dedekind's words, a constant corresponds or represents a thing, invariant in space and time, thus giving always the same number if measured properly.

An example of an invariable artifact is the PtIr-cylinder defining the kilogram. The melting point of a pure substance, the rest mass of an elementary particle, the velocity of a light beam in vacuum (corresponding mathematical symbol $c$ ) or the spin of an elementary particle (associated corresponding mathematical symbol $h$ ) are invariable phenomena given by nature. The corresponding number is said to be a constant of nature.

There are also important constants that correspond neither to an invariable artifact nor do they represent a naturally occurring, invariable phenomenon. Examples are the Avogadro constant $N_{\mathrm{A}}$, fixing the mole ( $\left\{N_{\mathrm{A}}\right\}$ specified atoms or molecules), or the number 157, fixing the barrel, the trading unit in the oil business (approximately 157 liter of oil). These constants are produced when needed according to the measuring procedures for the quantity involved; they are not given by nature and are not constants of nature.

In sciences, the term "constant" is usually used for both the quantity/number (free creation of the human mind) as well as for the invariable thing (phenomenon) that 
represents each other. However, one has to keep in mind that they are different things and must not be mixed up.

\section{Systems of units}

Measurability requires that all measurable quantities can be assigned unequivocally a number by measurement. Since our current understanding of the observable world does not yet allow a full mathematical description from first principles, a few numbers representing an observable, invariable phenomenon must be chosen by convention. These invariable phenomena with the corresponding, assigned numbers-i.e., these so-called constants-determine the system of units. These constants can no longer be measured, and they serve for adjusting/gauging the measuring instruments. Given all the laws of nature, only a few number of measurable constants can be fixed arbitrarily. Otherwise we would get an overdetermination that would lead to inconsistent measurement results (differing numbers for the same observable thing).

As the laws of nature, i.e., the functional dependencies among the measurable quantities do not depend on the units we use for measurement (the dependence between observable things is given by nature), it follows that the number of constants that must be assigned a value by convention is not dependent on the particular selection of the constants that fixes the system of units. With today's knowledge, the only condition on the constants fixing a system of units is that the constants allow to form a number for mass, time, length, an electrical quantity and temperature. That means if five well-selected constants are chosen that allow to build up values of the five mentioned quantities, all other values of measurable quantities are obtained by building multiples, submultiples and products of powers of these constants, utilizing the known laws of nature and the specific measurement procedures for the quantities involved. All other values are derived from the fixed constants that define the system of units. The constants of various systems of units based on natural constants are shown in [12]. A more comprehensive discussion on the principles of constructing a system of units and the constants determining the SI is given in [13].

As the constants fixing the SI-up to the kilogram-do not have the value 1 , the practical problem is to find suitable procedures for building up experimentally the units (phenomena/things representing the value 1) for each quantity, given the constants with their assigned values. In the official SI-Brochure [14], this process is called "mise en pratique".

In the years after the Convention of the Meter [1] of 1875 , the units for mass, length and time were given by artifacts with the arbitrarily assigned value 1 (prototypes of kilogram, meter), and the earth rotation-later the rotation of the earth around the sun-defining the unit of time. The constants that were fixed implicitly by convention with these units are easily identified. However, in 1948 the unit ampere (definition see Appendix) was introduced in the SI. The purpose of its definition is anything but clear to the uninformed reader. Neither a constant is mentioned that might help to define the system of units, nor is there any indication on how the unit, the "thing" representing the value 1 of the quantity "electrical current" should be realized. The key to these questions is found in the minutes of the 46th meeting of the CIPM of 1946 [15], where it was stated that the only purpose of the definition was to fix the constant in the general formula expressing the force between conductors, that the wording was chosen for its verbal simplicity and that the realization had to be effected according to the general laws in electromagnetism.

Obviously, the ampere "definition" defines a fictitious unit and does not contain any information on how the real unit had to be realized. As the minutes of this meeting are not available to the scientific public at large, any scientist is left alone to find out what the purpose of this wording might be, then to find out the constant that is buried in the wording, and finally to find out how experiments have to be designed in order to produce the real unit or other reference values of current (multiples and submultiples of the unit). The only clear information in the definition is that the name of the unit is "ampere". The real thing representing the value 1 of current is not defined. The wording of the "ampere definition" is rather an example of obscure writing, but not a definition. Unfortunately, in the current discussion on a possible redefinition of base units many proposals for possible wordings of base units are forwarded that are of a similar type as the ampere definition. Instead of clear definitions of the thing/unit representing the value 1 of the quantity, formulations are proposed with hidden constants that are fixed implicitly [16]. Such formulations serve nobody and should be discarded.

In any way, the essential part of the SI are not the base units, but the constants that define the system [13]. It is only nearly 60 years later that in the 8 th edition of the SI brochure [14] of 2006, the constants determining the SI are explicitly mentioned for the first time below the definitions of the base units. Experimentally, the measurement scales can be constructed consecutively starting from these known values.

For the reader who scrutinizes carefully the literature on units, it is not clear why in all other systems of units five constants are sufficient to fix the system, whereas in the SI seven constants are mentioned, one for each base unit. Checking the constants closer, it is easily recognized that only the first five constants fixed in the definitions for kilogram, meter, second, ampere and kelvin are constants 
corresponding/representing an observable, invariable phenomenon. The constants appended at the end of the definitions of the mole and candela (see below) are neither given by nature nor by a human artifact. There is no observable thing corresponding to these values. These constants are neither available for gauging a measuring instrument nor necessary for measuring the associated quantities. Nevertheless, the mole and the candela are called "base quantities" and are considered as having an own "dimension" [2-4].

\section{Base quantities, base units and dimensions}

In contrast to any other system of units, the SI introduces the concepts "base quantity", "base unit", and "dimension", the latter being defined on the basis of the concept "base quantity" [14]. Due to the fact that the base quantities contain a purely derived quantity like the candela and a quantity based on a number that can in principle simply be counted (the mole), it is clear that there exists no consistent logical foundation for the concepts "base quantity", "base unit", "derived quantity", "derived unit" and "dimension", as introduced in the SI. A base quantity is "base" by definition, and the terms "derived" and "dimension" have not the same meaning as in the mathematical literature. The lack of mathematically wellfounded concepts emerging from a literal interpretation of Maxwell's statement is highly confusing and prevents not only the general reader to get a clear understanding of the subject, but even the responsible bodies of the SI, as is demonstrated further by a phrase like the following, [14]:

Finally, it should be recognized that although the seven base quantities-length, mass, time, electric current, thermodynamic temperature, amount of substance, and luminous intensity - are by convention regarded as independent, their respective base units-the metre, kilogram, second, ampere, kelvin, mole, and candela-are in a number of instances interdependent.

Firstly, it is to say that dependency or independency between mathematical objects like quantities is not a question of convention, but only of verifiable mathematical facts. It is trivial to show that there are manifold functional dependencies between the so-called base quantities. Secondly, dependency between two units mean, that the second unit changes if the first unit undergoes a change. The quantities that are measured in these units then inevitably show changes as well, otherwise units and quantities would not correspond to each other (the mathematical thing "quantity" would not correspond to the observable thing "unit"). Thus, the interdependence between units and quantities measured in these units is inevitably the same. Such logical flaws in the official text on the SI is inacceptable and demonstrates the urgent need for a mathematically correct foundation of the SI, in which mixed constructs of numbers and units as forwarded under the "quantity calculus" have no place.

As a conclusion, the concept of dimension and dimensionality, based on the seven arbitrarily selected base quantities/units, is founded on the names/symbols of the units and has no mathematical foundation. Neglecting this fact leads to sometimes peculiar discussions, as for example on whether a quantity has dimension 1 or no dimension [17], or on the proposal to introduce the name "uno, symbol U" for the unit one [18]. As we have seen previously, a measurement unit is an observable thing. The authors of such a proposal had to explain what the thing is that should be called "uno". Such discussions show that there is a deep confusion between mathematical objects (quantity), observable objects (units) and the names and symbols used for describing them. There are results where the numbers of measurement are not expressed in one-or a combination-of the seven names/symbols appended to the "base units", as for example for plane angle or sound level, where the quantity stands for a ratio of measurable quantities. But to state that plane angle/sound level or their units should be "dimensionless" shows that observable things in nature and their corresponding quantities are confused with names/symbols of the things involved. Without two "dimensions" of length no plane angle is realized! These "dimensionless" quantities can be measured as well as any other "dimensional" quantity. This shows that names and symbols given to quantities and units for the sake of communication must not be confused with mathematical concepts.

Sticking to Dedekind's well-founded concept of numbers, the discussions on whether a quantity is dimensionless or not are meaningless. All values are pure numbers. When the number corresponds to a measurable quantity, this can be indicated in words or by appending the name or the symbol of the unit to the number. The interdependency between the quantities in turn is given by the laws of nature, and these laws are not dependent on the names and symbols appended to the units. The discussion on whether the units or quantities are dependent or interdependent, becomes obsolete. Thus, the functional dependency between the quantities modeling or representing the observable phenomena is the same in all systems of units. As a consequence, the so-called dimensionality of different systems of units [8] has no mathematical foundation [13]; it is based on the names or symbols appended to the numbers for the sake of communication.

The "independency" between mass, length, time, electricity and temperature is reflected by the need to assign 
five constants a value by convention, as explained earlier. Otherwise, not all quantities could be measured. Butremarkably enough - mass, time and length become functionally interdependent, if the Planck constant $h$ is assigned a fixed number by convention, as it is planned in the next redefinition of the unit of mass [19].

The mole and the candela-introduced in the SI as "base units"-must not be confounded with the units of the five quantities that are based on fixed constants that represent an observable phenomenon.

In chemistry, large numbers of atoms and molecules have to be handled in stoichiometrically correct ratios. This is easily done with the balance. The indication of the balance, divided by the atomic or molecular mass provides directly the number of atoms or molecules in counting units of $N_{\mathrm{A}}$, the Avogadro constant. The balance has the same function in chemistry as in a bank, where large numbers of coins are counted with balances. Assigning the Avogadro constant a fixed value would withdraw the chemist conceptually a most efficient counting device, the balance.

It is argued that, by assigning the Avogadro constant a fixed value, the uncertainty of the "fundamental" constants would be improved, as the gas constant $R$ and the faraday constant $F$ would become fixed numbers, too [16]. This would be true only if the Avogadro constant could be counted exactly. Due to its staggering size, this is not feasible. Thus, the "accuracy" achieved by assigning the Avogadro constant a fixed value would only be a fictitious one on paper. In measurement, the uncertainty in realizing macroscopic amounts of substances cannot be avoided. Decoupling the Avogadro constant from mass by assigning it a fixed value would make it useless for measurement and uncertainty evaluations. The constant would simply lose its meaning and become scientifically irrelevant. A more comprehensive discussion of the subject is given in $[11,20$, 21].

A further conceptual mistake of this proposal is that the Avogadro constant is set on the same level as the other five constants fixing the SI. This constant, however, is an arbitrary number based on an arbitrary selection of carbon12 as reference for the relative atomic mass scale. In nature, there is nowhere a thing representing $N_{\mathrm{A}}$, thus is not a "fundamental constant of nature" as claimed in [16]. Even more amazing is the fact that in the official definition of the mole (see Appendix), the Avogadro constant is not mentioned (same situation as in the ampere definition) and logic is turned upside down. It is claimed that from the definition follows that the molar mass of carbon 12 is exactly $12 \mathrm{~g}$ per mole, $M\left({ }^{12} \mathrm{C}\right)=12 \mathrm{~g} / \mathrm{mol}$ [14]. As can easily be checked by reading the wording carefully, the selection of carbon-12 is the assumption, the conclusion of the definition is the Avogadro number that is fixed implicitly. The assumption fixes the relative mass scale of atoms and molecules for enabling the counting of atoms and molecules with the balance. This example shows once more how confusing definitions with implicitly fixed constants are, even for the specialists working on conceptual matters of units!

The candela (definition see Appendix) in turn is a purely derived unit, given the radiometric units and the measured sensitivity of the human eye on the visible spectrum of radiation. Again, for the value appended to the definition there is neither a corresponding artifact nor any thing given by nature.

It is further expected that a definition of a term would determine the term entirely. However, according to the official text, the candela is defined for a frequency of $540 \times 10^{12} \mathrm{~Hz}$ only. The most important part of photometry, the measured standard luminosity function $V(\lambda)$ which describes the sensitivity of the eye over the full spectrum of human vision is not even mentioned. This function is known to be dependent on the level and on the angle of the incident light on the eye. The absence of such essential influence parameters in a definition makes it of little benefit for the scientist. The definition is incomplete and does not fix the unit over its range of application.

It is apparent that assigning an own name and "dimension" to a number of things or a derived thing is not a basis for a scientifically sound theory.

It is a strange fact that metrologists in the surroundings of the Meter Convention have developed over the last 100 years-possibly driven by the literal interpretation of Maxwell's statement cited earlier-a terminology [8] that has never been adopted by the great physicists that have influenced the education of physicists over generations (Sommerfeld, Landau, Feynman and others). Terms like "system of quantities", "base and derived unit", "quantity calculus" that are based on unit names and unit symbols are neither necessary nor mathematically well founded. They convey a misleading picture of the observable world and lead to logical flaws and contradictions in the mathematical description, as shown. They should be avoided.

\section{Outlook}

Most recently, the CCU has forwarded another proposal for a redefinition of the base units [19]. The SI is introduced as follows:

It is defined by specifying the values of seven base units, and then treating all other units as derived units whose values are given as products of powers of the base units without including any numerical factors, following the corresponding relations between the quantities involved. The present definitions of the 
seven base units are made in terms of the values of seven fundamental constants that are believed to be true invariants throughout time and space, available to anyone, anywhere, at any time, who wishes to realize and make use of the values of the units to make measurements.

The new definitions of the base units shall be of the "explicit-constant" type, that is, a definition in which the unit is defined indirectly by specifying explicitly an exact value for a well-recognized fundamental constant. It is further claimed that these constants were "invariants of nature".

The proposed wording is as follows (the symbol $\mathrm{X}$ represents additional digits to be added at the time that these revised definitions would be adopted):

- The second, s, is the unit of time; its magnitude is set by fixing the numerical value of the ground state hyperfine splitting frequency of the cesium 133 atom to be equal to exactly 9192631770 when it is expressed in the unit $\mathrm{s}^{-1}$, which is equal to hertz.

- The meter, $\mathrm{m}$, is the unit of length; its magnitude is set by fixing the numerical value of the speed of light in vacuum to be equal to exactly 299792458 when it is expressed in the unit $\mathrm{m} \mathrm{s}^{-1}$.

- The kilogram, $\mathrm{kg}$, is the unit of mass; its magnitude is set by fixing the numerical value of the Planck constant to be equal to exactly $6.62606 \mathrm{X} \times 10^{-34}$ when it is expressed in the unit $\mathrm{s}^{-1} \mathrm{~m}^{2} \mathrm{~kg}$, which is equal to $\mathrm{J} \mathrm{s}$.

- The ampere, A, is the unit of electric current; its magnitude is set by fixing the numerical value of the elementary charge to be equal to exactly $1.60217 \mathrm{X} \times$ $10^{-19}$ when it is expressed in the unit s $\mathrm{A}$, which is equal to $\mathrm{C}$.

- The Kelvin, $\mathrm{K}$, is the unit of thermodynamic temperature; its magnitude is set by fixing the numerical value of the Boltzmann constant to be equal to exactly $1.3806 \mathrm{X} \times 10^{-23}$ when it is expressed in the unit $\mathrm{s}^{-2} \mathrm{~m}^{2} \mathrm{~kg} \mathrm{~K} \mathrm{~K}^{-1}$, which is equal to $\mathrm{J} \mathrm{K}^{-1}$.

- The mole, mol, is the unit of amount of substance of a specified elementary entity, which may be an atom, molecule, ion, electron, any other particle or a specified group of such particles; its magnitude is set by fixing the numerical value of the Avogadro constant to be equal to exactly $6.02214 \mathrm{X} \times 10^{23}$ when it is expressed in the unit $\mathrm{mol}^{-1}$.

- The candela, cd, is the unit of luminous intensity in a given direction; its magnitude is set by fixing the numerical value of the luminous efficacy of monochromatic radiation of frequency $540 \times 10^{12} \mathrm{~Hz}$ to be equal to exactly 683 when it is expressed in the unit $\mathrm{s}^{3} \mathrm{~m}^{-2} \mathrm{~kg}^{-1} \mathrm{~cd} \mathrm{sr}$, or cd $\mathrm{sr} \mathrm{W}^{-1}$, which is equal to $\operatorname{lm} \mathrm{W}^{-1}$.
With these wordings, all the shortcomings and drawbacks commented previously are perpetuated or even increased. The most important are the following:

- The "definitions" are not understandable; not to the scientist, let alone to the general reader.

- Names and symbols of the units are confused once more with the units (the second is the unit of time, ...).

- The real units, the measurable things representing the value 1 of the quantity, are not defined. The scientist is let alone to find out how experiments have to be designed for realizing the real units.

- Simply wrong is the association of a unit with a single constant. As most "base units" are dependent on each other, stating only one constant in the definition does not determine those units fully. For example, the meter could be anything according to the given definition. Doubling the duration of the second would double the meter as well. As the second is not specified in the wording, the definition is incomplete. These "definitions" are complete only in their totality, that means if all definitions are available at the same time. However, under this condition the "definitions" are trivial, as all measured values are such that the constants defining the system of units have their assigned values.

- The definitions convey the idea that the "base units" would determine the system of units, the derived units being derived from them. Obviously, this is wrong. Without the availability of the derived real units joule and watt the kilogram, kelvin and candela cannot be realized. This means that the definitions are circular: Base units are defined in terms of derived units that are said to be derived from the base units. Such logical flaws are not acceptable in the official text on the SI!

- The constants given in the mole and candela definitions are still not "invariants of nature". There is nowhere a thing in nature corresponding to these constants. To call the Avogadro constant a true invariant throughout time and space is about the same as one would claim that the dozen is a true invariant throughout time and space.

All these contradictions and drawbacks can be avoided if the well-founded notions as provided by the mathematicians [5] are applied properly:

Mixed constructs of numbers and names or symbols of units have no justification. Observable phenomena and units used in measurement, their corresponding mathematical objects (quantities, numbers), and the names and symbols used for the linguistic description of both must be kept clearly apart.

Notions that cannot be defined clearly like "base/ derived quantity", "base/derived units" and "system of 
quantities" must be avoided. There is no need for them, the great theoretical physicists of the last century have never used these notions. They are in contradiction with mathematical facts, as has been shown earlier.

Along these guidelines, the SI could be presented with the constants given earlier in a clear and logically concise way as follows:

- Names and symbols of the units having an own name must be declared as what they are: Terms of the normal language. They must be listed in a separate table and not stated implicitly as units in "definitions".

- Then, as the most important part of any system of units, the constants that determine the system must be stated. These constants have by convention an assigned value and correspond to an invariable phenomenon given by nature.

- Normal numbers or measured numbers used in some units having no correspondence in nature must not be mixed up with these system-relevant constants of nature and should be given separately. Among others these are the Avogadro constant for chemical measurements, the reference radiation power for photometrical measurements and the reference sound level for sound measurements.

- And finally, as the SI is a practical system of units, it is necessary to indicate how the real units or scales of measurement are built up in logically consecutive steps for the most important quantities, starting from the given constants, and without referring to units not introduced previously. This prevents circular definitions as in the aforementioned wordings.

Such a mise-en-pratique must include all quantities that follow naturally from the given constants, and cannot be restricted to the so-called base units. Hence, given for example Planck's constant $h$ and elementary charge $e$, it is straight forward to introduce first the "derived" units coulomb, volt and ohm, the latter being realized by the wellknown quantum experiments utilizing the Josephson and the quantum Hall effect. With these established electrical units and scales of measurement, the unit of current, the ampere, as well as the units of energy and power-joule and wattare easily realized. It's only when these "derived" units are available, that a mise-en-pratique or realization of the real unit of mass via the watt experiment [22] is possible.

Liberated from ill-defined concepts like base and derived quantity/unit, other important units like those of the luminous intensity (cd), amount of substance (mol) or sound level $(\mathrm{dB})$ could be smoothly added to the units derived from the five fixed constants of nature given earlier.

A presentation of the International System of Units (SI) along these guidelines would greatly improve the understandability of the basic concepts underlying any systems of units and avoid the notions that were shown to be in contradiction with well-established mathematical concepts like numbers and variables/quantities. Finally, it is the duty and task of an international body like the Meter Convention to introduce and maintain terms and notions that are in agreement with proved and established science $[21,23]$.

\section{Conclusions}

The International System of Units (SI) as developed under the Meter Convention has introduced in the last century terms and concepts that are in contradiction with wellfounded mathematical notions and that were never used by the famous theoretical physicists of the twentieth century. In particular, the often used dogma of a quantity as being the product of a number and a unit [8] is a source of contradictions and logical flaws. Most popular terms like "system of quantities", "base quantity", "derived quantity", "base unit" and "derived unit" have no logical sound foundation and lead continuously to confusion and contradiction as well.

It is shown that this confusion can be avoided if the well-established mathematical notions are applied. There, the values of all quantities are the well-known numbers as defined in the mathematical literature and are not combined with names or symbols of units. Using mathematical notions, purely abstract concepts (quantity, number) and the observable things (units/phenomena) are logically well kept apart. Names and symbols of units are clearly identified as what they are: Elements of normal language that serve in communication. They have no mathematical properties, neither magnitude nor dimension. These mathematically consistent notions as developed and forwarded by the famous mathematicians like Richard Dedekind [5] in the nineteenth century lead to a better understandability of natural sciences and avoid logical flaws and lengthy discussion provoked by non-mathematical and badly founded terms and concepts. Finally, it is briefly sketched how the SI could be presented avoiding the misleading notions of "base" and "derived" units.

Acknowledgments I express my highest appreciation to the editor for the encouragement and support to forward views that are not known or accepted in parts of the scientific community. Unfortunately, this open-minded position is anything but self-evident even in sciences!

Open Access This article is distributed under the terms of the Creative Commons Attribution Noncommercial License which permits any noncommercial use, distribution, and reproduction in any medium, provided the original author(s) and source are credited. 


\section{Appendix}

\section{Ampere}

The ampere is that constant current which, if maintained in two straight parallel conductors of infinite length, of negligible circular cross section, and placed 1 meter apart in vacuum, would produce between these conductors a force equal to $2 \times 10^{-7}$ newton per meter of length.

\section{Mole}

1. The mole is the amount of substance of a system which contains as many elementary entities as there are atoms in $0.012 \mathrm{~kg}$ of carbon 12; its symbol is "mol".

2. When the mole is used, the elementary entities must be specified and may be atoms, molecules, ions, electrons, other particles, or specified groups of such particles.

It follows that the molar mass of carbon 12 is exactly $12 \mathrm{~g}$ per mole, $M\left({ }^{12} \mathrm{C}\right)=12 \mathrm{~g} / \mathrm{mol}$.

\section{Candela}

The candela is the luminous intensity, in a given direction, of a source that emits monochromatic radiation of frequency $540 \times 10^{12} \mathrm{~Hz}$ and that has a radiant intensity in that direction of $1 / 683 \mathrm{~W}$ per steradian.

It follows that the spectral luminous efficacy for monochromatic radiation of frequency of $540 \times 10^{12} \mathrm{~Hz}$ is exactly 683 lumens per watt, $K=683 \mathrm{~lm} / \mathrm{W}=683 \mathrm{~cd} \mathrm{sr} / \mathrm{W}$.

\section{References}

1. Convention of the Meter. Accessed Dec 2010. http://www.bipm. org/en/convention

2. BIPM, IEC, IFCC, ISO, IUPAC, IUPAP, OIML (1993) International vocabulary of basic and general terms in metrology, 2nd edn. ISO, Geneva

3. BIPM, IEC, ISO, OIML (1983) International vocabulary of basic and general terms in metrology. ISO, Geneva

4. BIPM, IEC, IFCC, ILAC, ISO, IUPAC, IUPAP, OIML (2008) International vocabulary of metrology-basic and general concepts and associated terms (VIM), 3rd edn JCGM vol 200. Accessed Dec 2010. http://www.bipm.org/vim

5. Dedekind R (1893) Was sind und was sollen die Zahlen? Friedrich Vieweg und Sohn, Braunschweig, 1893 [English translation: The nature and meaning of numbers. Accessed Dec 2010. http://www.archive.org/details/essaysintheoryof00dedeuoft]

6. Sibley TQ (2009) The foundations of mathematics. Wiley

7. Reinhardt F, Soeder H (1974) dtv-Atlas zur Mathematik: Band 1. Deutscher Taschenbuch Verlag, München

8. de Boer J (1995) On the history of quantity calculus and the international system. Metrologia 31:405-429

9. Feynman RP (1966) The Feynman Lectures on Physics, vol 1. Addison-Wesley Publishing Company, fourth printing $\mathrm{p}$ 4-1

10. de Bièvre P, Price G (2009) Simple principles for metrology in chemistry: identifying and counting. Accred Qual Assur 14: 295-305

11. Draft Chapter 2 for SI Brochure, Contribution CIPM2009-47 of CCU to the 98th meeting of CIPM (2009). BIPM, Sèvres

12. Natural units. Accessed Dec 2010. http://en.wikipedia.org/wiki/ Natural_units

13. Feller $\mathrm{U}$ A constructional approach to the international system of units (SI). Contribution CCE/97-3 to the 21st meeting of the Comité Consultatif d'Electricité. BIPM, Sèvres. Accessed Dec 2010. http://www.metas.ch/root_legnet/Web/Dokumentation/ Publikationen/Wissenschaftspublikationen/General/PDF/Approach_ to_SI.pdf

14. The International System of Units (SI), 8th edn, 2006. Accessed Dec 2010. http://www.bipm.org/utils/common/pdf/si_brochure_ 8_en.pdf

15. Bureau International des Poids et Mesures (1946). In: Proceedings-Verb Com International Poids et Mesures, vol 20, p 133

16. Mills IM et al (2006) Redefinition of the kilogram, ampere, kelvin and mole: a proposed approach to implementing CIPM recommendation 1 (CI-2005). Metrologia 43:227

17. Giacomo P (1995) Sans dimension ou de dimension un? Metrologia 32:311

18. Quinn TJ, Mills JM (1998) The use and abuse of the terms percent, parts per million and part in $10^{\mathrm{n}}$. Metrologia 35:807

19. Draft Chapter 2 for SI Brochure, document CCU/10-00DraftCh2PDF of the 20th meeting of CCU (2010). BIPM, Sèvres. Accessed Dec 2010. http://www.bipm.org/cc/CCU/Allowed/20/Watermark_SI_ Brochure_Draft_Ch2_29Sep10.pdf

20. Andres HP et al (2009) No rationale for a redefinition of the mole. Chimia 63:616

21. Price $G$ (2010) Failures of the global measurement system. Part 1: the case of chemistry. Accred Qual Assur 15:421

22. Schwitz W et al (2004) Towards a new kilogram definition based on a fundamental constant. C R Phys 5:881

23. Price G (2010) Failures of the global measurement system. Part 2: institutions, instruments and strategy. Accred Qual Assur 15:477 